\title{
SUSCEPTIBILITY TESTING OF PSEUDOMONAS AERUGINOSA BY THE VITEK 2 SYSTEM: A COMPARISON WITH ETEST RESULTS
}

\author{
V. Saegeman ${ }^{1 \mathrm{a}}$, P. Huynen ${ }^{3}$, J. Colaert ${ }^{2}$, P. Melin ${ }^{3}$, J. Verhaegen ${ }^{1}$
}

Key-words: susceptibility testing, Pseudomonas aeruginosa, Vitek 2 system, Etest

\begin{abstract}
P. aeruginona infections need accurate antimicrobial susceptibility data, as treatment mainly relies on antibiotic efficiency in debilitated patients. Vitek 2, a popular automated susceptibility testing method, was compared with Etest to assess its reliability on 150 Belgian P. aeruginonas isolates.

Vitek 2 and Etest exhibited a high degree of concordance, but some discrepancies in clinical category were evident for cefepime (high minor and borderline very major error rate) and for piperacillin/tazobactam (high very major error rate). Vitek 2 appears to yield valuable information to the clinician concerning the antimicrobials amikacin, ceftazidime, ciprofloxacin and meropenem, in the setting of pseudomonas infection. For cefepime and piperacillin/tazobactam, a confirmatory testing by means of disk diffusion is worth considering.
\end{abstract}

1a UZ Gasthuisberg, KULeuven, Dept. Microbiologie, Herestraat 49, 3000 Leuven, Aspirant Researcher of the Fund for Scientific Research-Flanders

1 UZ Gasthuisberg, KULeuven, Dept. Microbiologie, Herestraat 49, 3000 Leuven

2 AZ Groeninge Maria's Voorzienigheid, Dept. Microbiologie, Looftstraat 43, 8500 Kortrijk

3 Centre Hospitalier Universitaire de Liège, Domaine Universitaire du Sart-Tilman, Univ. de Liège, Dépt. Microbiologie, Bâtiment B 35, 4000 Liège 1

Address for correspondence:

Dr. V. Saegeman,

Yzermolenstraat 152

Bus 13, 3001 Heverlee

E-mail: veroniek.saegeman@uz.leuven.ac.be

\section{INTRODUCTION}

Pseudomonas (P.) aeruginosa infections pose several problems to the clinician. ${ }^{1,2}$ Most often, they occur in debilitated patients with severe underlying disease and disturbance of the host defence mechanisms. ${ }^{3,4}$ Moreover, even sensitive strains usually exhibit MIC values close to the susceptibility breakpoint for any given antipseudomonal antimicrobial. Therefore, when reporting the in vitro susceptibility pattern of a P. aeruginosa strain to the clinician, it is of utmost importance for it to be accurate. Moreover, most patients will largely depend, rather than on their own defence system, on the antimicrobial activity of the agent(s) administered and on supportive care.

In Belgium, several teaching and large general hospital laboratories make use of the Vitek 2 automated system (bioMerieux Vitek, Hazelwood, USA) for species identification and antimicrobial susceptibility determination. Several accuracy evaluations of Vitek 2 have been published. ${ }^{2,4-14}$

One of the benefits of this rapid test is provision of test information sooner so that changes in antibiotic therapy, when indicated, can be implemented more quickly. ${ }^{15}$ Vitek 2 offers a standardized method ideally suited to laboratories lacking familiarity with, myriad resistance mechanisms and/or those not testing an appropriate number, or range, of antibiotics to detect resistant phenotypes using interpretative reading of MICs. ${ }^{6}$ The Vitek Advanced Expert System comprises a database of MIC distribution for different combinations of antibiotics and prevalent resistance mechanisms in different species, together with a series of algorithms. Both, unlikely combinations of phenotype and species as well as inferred mechanisms predicting 
clinical resistance to drugs to which bacteria appeared susceptible at breakpoint are highlighted. ${ }^{7}$ Although this system can provide results within $6 \mathrm{~h}$, there are shortcomings. They can for instance misidentify certain clinically important mechanisms of antimicrobial resistance. ${ }^{8}$ Cell-wall active compounds like cefepime, ceftazidime, meropenem and piperacillin/tazobactam, used with an inappropriately high inoculum, cause higher MICs with the Vitek system when $P$. aeruginosa is tested. ${ }^{5,6}$ Vitek's automated susceptibility testing results have previously shown to be very misleading for imipenem when testing $P$. aeruginosa. ${ }^{16}$ Other drawbacks of automated systems are related to the narrow range of antibiotic concentrations included in the antimicrobial cards, which precludes the detection of resistances expressed at a low level. ${ }^{8}$ Moreover, patients with cystic fibrosis frequently have a unique $P$. aeruginosa phenotype including the presence of mucoid exopolysacccharide and relatively slow growth rates in the laboratory, features which may make standardized performance and interpretation of antimicrobial susceptibility testing difficult. ${ }^{11}$

This report compares Vitek 2 with Etest (AB Biodesk, Solna Sweden) susceptibility results using 150 clinical $P$. aeruginosa isolates from 3 large-sized hospitals.

In case the overall very major error rate (susceptible result with Vitek 2 and resistant with Etest) was equal to or exceeded $1.5 \%$ for a specific antibiotic (occurring for piperacillin/tazobactam and cefepime), the strains with this error occurring in at least two of the 3 participating hospitals were further analysed. At one centre, the Sensititre system (Trek Diagnostic Systems Inc, West Sussex, UK, providing quantitative susceptibility results in a dried panel format) was applied on these strains in order to reach a consensus result.

\section{MATERIALS AND METHODS}

Each centre collected 50 consecutive non-duplicate clinical $P$. aeruginosa strains, subsequently forwarded its strains to the other two participating centres, each finally disposing of 150 isolates to be tested. The following antibiotics were assessed: amikacin, cefepime, ceftazidime, ciprofloxacin, meropenem and piperacillin/tazobactam.

Results were expressed in terms of clinical (Susceptible/Intermediate/Resistant) categories. For the whole collection, comparisons were made between both methods, as well as between centres considered separately.

Susceptibility testing with the Vitek 2 system was performed with standard European AST-N020 cards according to the following method. Strains were subcultured, subsequently grown in ambient air for $18-24 \mathrm{~h}$ at $35^{\circ} \mathrm{C}$ on Tryptic Soy Agar containing 5\% horse blood. Suspensions of these cultures were made in $0,45 \%$ saline, adjusted to the $0,5 \mathrm{McFarland}$ turbidity standard, and used to fill the test cards for the Vitek 2 instrument applied according to the manufacturer's instructions.

The Vitek 2 system automatically measures a turbidity signal for each test well containing an antibiotic, every $15 \mathrm{~min}$ for up to $18 \mathrm{~h}$. These data are used to generate growth curves and by comparison with a control, the MIC of each antibiotic is estimated.

Each agent is included in the AST-N020 test card at two to four different concentrations: amikacin: 2-64 $\mu \mathrm{g} / \mathrm{ml}$, cefepime and ceftazidime: $1-64 \mu \mathrm{g} / \mathrm{ml}$, ciprofloxacin: $0.25-4 \mu \mathrm{g} / \mathrm{ml}$, meropenem: $0.25-16 \mu \mathrm{g} / \mathrm{ml}$, piperacillin/tazobactam: 4-128 $\mu \mathrm{g} / \mathrm{ml}$.

All results outside this range are reported as either the lowest or highest level available.

Susceptibility testing with Etest strips was performed according to following method. Etests were run in parallel with MIC determinations on the Vitek 2 system. The same subculture was used for both methods. A suspension of several well isolated $P$. aeruginosa colonies was prepared from an overnight grown blood agar plate, with a turbidity adjusted to visually match that of the $0,5 \mathrm{McF}$ arland turbidity standard. The six Etest strips, each containing a different antimicrobial agent, were placed radially on the surface of a large round Mueller-Hinton agar plate inoculated with the bacterial suspension. After incubation for 18 to $24 \mathrm{~h}$ in ambient air at $35^{\circ} \mathrm{C}$, the MIC was read directly from a scale on top of the strip at the point where the edge of the inhibition ellipse intersects the side of the strip.

Etest generates MIC values from a continuous scale (results in two-fold dilutions) and extending either from 0.016 to $256 \mu \mathrm{g} / \mathrm{ml}$ (ceftazidime, amikacin, cefepime, piperacillin/tazobactam), or from 0.002 to 32 $\mu \mathrm{g} / \mathrm{ml}$ (ciprofloxacin and meropenem).

Breakpoints for susceptibility category determination (Susceptible/Intermediate/Resistant) were those recommended by the NCCLS guidelines. ${ }^{17}$

Results were expressed in terms of MIC $(\mu \mathrm{g} / \mathrm{ml})$, translated into clinical categories $(\mathrm{S} / \mathrm{I} / \mathrm{R})$ and differences between both methods were defined as: 
- agreement in clinical category or ACC (result identical with both methods)

minor error: $\mathrm{S}$ with Etest and I with Vitek or vice versa, $\mathrm{R}$ with Etest and I with Vitek or vice versa

major error: $\mathrm{S}$ with Etest and $\mathrm{R}$ with Vitek

very major error: $\mathrm{R}$ with Etest and $\mathrm{S}$ with Vitek

When for a certain strain, a very major error was registered in at least two of the centres, Sensititre was performed as the reference broth microdilution method (NCCLS). The consensus result was defined by the test corresponding with Sensititre, conform to Chandler's performance study of the Vitek System (software version VTKR07.01). ${ }^{12}$

Sensititre plate susceptibility testing was performed according to the following method. In demineralised water, 3 to 5 P. aeruginosa colonies from an overnight grown blood agar plate were suspended and adjusted to reach the $0.5 \mathrm{McF}$ arland turbidity standard. $10 \mu \mathrm{l}$ of the suspension was transferred into a tube of $11 \mathrm{ml}$ cation-adjusted Mueller-Hinton broth. After vortexing, $50 \mu \mathrm{l}$ of this concentration was inoculated (using a multi-pipettor) into each of the wells. The plate was covered with an adhesive seal and incubated in ambient air at $35^{\circ} \mathrm{C}$ for $18-24 \mathrm{~h}$. Growth appears as turbidity or as a deposit of cells at the bottom of a well. The MIC is recorded as the lowest concentration of antibiotic inhibiting visible growth.

The dilution ranges available on the Sensititre plates are: amikacin: 4-32 $\mu \mathrm{g} / \mathrm{ml}$, cefepime: $2-16 \mu \mathrm{g} /$ ml, ceftazidime: $1-16 \mu \mathrm{g} / \mathrm{ml}$, ciprofloxacin: 0.25-2 $\mu \mathrm{g}$ / $\mathrm{ml}$, piperacillin/tazobactam: 8/4-64/4 $\mu \mathrm{g} / \mathrm{ml}$.

\section{RESULTS}

\section{According to clinical categories.}

Overall, the percentage of agreement in clinical category (ACC) between both methods was $91.15 \%$. There were $8.05 \%$ minor errors, $0.57 \%$ major errors, $1.57 \%$ very major errors and a total error rate of $8.85 \%$.

Table 1, row 2-4 gives an overview of the comparative test results in all three centres analysed collectively. For every antibiotic tested, the table shows the percentages of resistant, intermediate and susceptible strains with both methods. The last column represents the mean percentages of all antibiotics together.

The resistance of $P$. aeruginosa varied from less than $10 \%$ (amikacin with Etest, cefepime and mero- penem with Vitek) to almost $30 \%$ (ciprofloxacin, both methods). There were some differences between both methods.

Every discrepancy in clinical category registered in a centre was counted as one error, independently of the result in the other two centres.

Table 1, row 5-9 shows the concordance between both methods, for each antibiotic, expressed as either the rate of agreement in clinical category, or the error rate (minor, major, very major and total). The last column again shows the mean rate per error category for all antibiotics pooled.

Cefepime excepted, ACC exceeded 90\% for all antimicrobial agents tested. Combined minor-major error rates are excessive $(>7 \%)$ for amikacin, cefepime and ceftazidime. For cefepime, the low ACC was due to a high rate of minor errors and $1.50 \%$ very major errors. No antibiotic exhibited a large number of major errors (>3\%), but piperacillin/tazobactam had an unacceptably high frequency $(6.20 \%)$ of very major errors.

Piperacillin/tazobactam and cefepime, the two antibiotics with the highest rate of very major errors were further analysed with the Sensititre system.

Table 2 represents the results of Vitek 2, Etest and Sensititre method performed on the seven strains with a very major error between Vitek 2 and Etest in at least two of the three centres.

Compared with Sensititre, Vitek 2 also showed very major errors for piperacillin/tazobactam in 6 of the 7 strains with very major errors between Vitek2/Etest. Etest seemed to fall in the major error category just once with piperacillin/tazobactam. For the only very major error between Vitek 2 and Etest with cefepime, Sensititre gave consent to Etest.

\section{According to the individual centres.}

Table 3 describes the comparison between the 3 centres, taken separately, in terms of clinical categories. Note that results have been calculated, using the same range distribution for both test methods.

For all antibiotics pooled together, the distribution of errors in each of the error categories was highly concordant between the three centres. One noticed a slightly increased percentage of major errors in one centre.

Table 4 describes the pattern of differences in results expressed as clinical categories (S/I/R) between both testing methods in all of the three centres: same error in all three, variable in all three, discrepancy only in one laboratory. All other strains tested, yielded fully concordant results. 
Table 1. Row 2-4: qualitative test results with Vitek $2(\mathrm{~V})$ and Etest (E), expressed as percentage of total; row 5-9: concordance between both methods.*

\begin{tabular}{|c|c|c|c|c|c|c|c|c|c|c|c|c|c|c|}
\hline \multirow[b]{2}{*}{$\mathrm{R}$} & \multicolumn{2}{|c|}{\begin{tabular}{l}
\multicolumn{2}{c}{ Amikacin } \\
$\mathrm{V}$ \\
\\
\end{tabular}} & \multicolumn{2}{|c|}{\begin{tabular}{l} 
Cefepime \\
$\mathrm{V}$ \\
\multicolumn{1}{c}{$\mathrm{E}$}
\end{tabular}} & \multicolumn{2}{|c|}{$\begin{array}{l}\text { Ceftazidime } \\
\mathrm{V} \quad(\%)\end{array}$} & \multicolumn{2}{|c|}{\begin{tabular}{|ccc}
\multicolumn{2}{|c}{ Ciprofloxacin } \\
$\mathrm{V}$ & & $\mathrm{E}$ \\
& $(\%)$ & \\
\end{tabular}} & \multicolumn{2}{|c|}{$\begin{array}{l}\text { Meropenem } \\
\mathrm{V} \quad \mathrm{E} \\
\end{array}$} & \multicolumn{2}{|c|}{\begin{tabular}{l} 
Piperacillin/ \\
Tazobactam \\
V \\
\multicolumn{1}{c}{ E }
\end{tabular}} & \multicolumn{2}{|c|}{$\begin{array}{cc}\text { V Mean } & \\
& \text { E }\end{array}$} \\
\hline & 11.75 & 8.55 & 9.62 & 15.63 & 13.89 & 14.13 & 27.14 & 28.27 & 8.33 & 10.28 & 11.97 & 17.95 & 13.78 & 15.80 \\
\hline I & 4.06 & 5.34 & 11.97 & 11.35 & 11.54 & 5.57 & 7.91 & 5.35 & 6.20 & 4.71 & - & - & 6.94 & 5.39 \\
\hline S & 84.19 & 86.11 & 78.42 & 73.02 & 74.57 & 80.30 & 64.96 & 66.38 & 85.47 & 85.01 & 88.03 & 82.05 & 79.27 & 78.81 \\
\hline $\mathrm{ACC}$ & \multicolumn{2}{|c|}{91.67} & \multicolumn{2}{|c|}{84.58} & \multicolumn{2}{|c|}{90.36} & \multicolumn{2}{|c|}{93.15} & \multicolumn{2}{|c|}{93.58} & \multicolumn{2}{|c|}{93.59} & \multicolumn{2}{|c|}{91.15} \\
\hline Minor error & \multicolumn{2}{|c|}{7.26} & \multicolumn{2}{|c|}{13.71} & \multicolumn{2}{|c|}{8.15} & \multicolumn{2}{|c|}{6.21} & \multicolumn{2}{|c|}{4.92} & \multicolumn{2}{|c|}{-} & \multicolumn{2}{|c|}{8.05} \\
\hline Major error & \multicolumn{2}{|c|}{0.85} & \multicolumn{2}{|c|}{0.21} & \multicolumn{2}{|c|}{1.28} & \multicolumn{2}{|c|}{0.43} & \multicolumn{2}{|c|}{0.43} & \multicolumn{2}{|c|}{0.21} & \multicolumn{2}{|c|}{0.57} \\
\hline $\begin{array}{c}\text { Very major } \\
\text { error }\end{array}$ & \multicolumn{2}{|c|}{0.22} & \multicolumn{2}{|c|}{1.50} & \multicolumn{2}{|c|}{0.21} & \multicolumn{2}{|c|}{0.21} & \multicolumn{2}{|c|}{1.07} & \multicolumn{2}{|c|}{6.20} & \multicolumn{2}{|c|}{1.57} \\
\hline Total error & \multicolumn{2}{|c|}{8.33} & & & 9. & & 6. & & 6. & & & & 8. & \\
\hline
\end{tabular}

*Calculations done after the ranges of both tests had been rendered identical; i.e. all Etest results below or beyond the Vitek range have been drawn level to the lowest or highest available Vitek dilution, respectively.

Table 2. Comparison Vitek 2 (V), Etest (E) and Sensititre (S) for strains with an excessive rate of very major errors (in at least two centres) between Vitek 2 and Etest.

\begin{tabular}{|c|c|c|c|}
\hline $\begin{array}{c}\text { Strain } \\
\text { number }\end{array}$ & \multicolumn{3}{|c|}{$\begin{array}{c}\text { Piperacillin/tazobactam } \\
(64-128 \mu \mathrm{g} / \mathrm{ml})\end{array}$} \\
\hline & $\mathrm{V}$ & $\mathrm{E}$ & $\mathrm{S}$ \\
\hline 27 & $64(\mathrm{~S})$ & $>256(\mathrm{R})$ & $>64(\mathrm{R})$ \\
\hline 74 & $64(\mathrm{~S})$ & $>256(\mathrm{R})$ & $>64(\mathrm{R})$ \\
\hline 81 & $64(\mathrm{~S})$ & $>256(\mathrm{R})$ & $64(\mathrm{~S})$ \\
\hline 43 & $64(\mathrm{~S})$ & $128(\mathrm{R})$ & $>64(\mathrm{R})$ \\
\hline 50 & $64(\mathrm{~S})$ & $128(\mathrm{R})$ & $>64(\mathrm{R})$ \\
\hline 10 & $64(\mathrm{~S})$ & $>256(\mathrm{R})$ & $>64(\mathrm{R})$ \\
\hline 18 & $64(\mathrm{~S})$ & $>256(\mathrm{R})$ & $>64(\mathrm{R})$ \\
\hline & & Cefepime \\
\hline & $8(\mathrm{~S})$ & $32(\mathrm{R})$ & $>16(\mathrm{R})$ \\
\hline
\end{tabular}

The same error in all three labs appeared once with amikacin and twice with cefepime, ceftazidime, ciprofloxacin and piperacillin/tazobactam.

Variable patterns were observed between 1 and 8 times with all antibiotics tested.

A discrepancy limited to one single laboratory appeared between 12 and 33 times, a phenomenon which did not occur for ceftazidime.
Table 3. Comparison of the results between the 3 centres, expressed as clinical categories.

\begin{tabular}{|c|c|c|c|c|}
\hline & $\begin{array}{c}\text { Centre 1 } \\
\text { (in \%) }\end{array}$ & $\begin{array}{c}\text { Centre 2 } \\
\text { (in \%) }\end{array}$ & $\begin{array}{c}\text { Centre 3 } \\
\text { (in \%) }\end{array}$ & $\begin{array}{c}\text { Average * } \\
\text { (in \%) }\end{array}$ \\
\hline ACC & 91.24 & 91.08 & 91.13 & 91.15 \\
\hline Minor error & 8.23 & 8.02 & 7.89 & 8.05 \\
\hline Major error & 0.21 & 1.06 & 0.44 & 0.57 \\
\hline Very major error & 1.69 & 1.17 & 1.86 & 1.57 \\
\hline Total error * & 10.13 & 10.25 & 10.19 & 10.19 \\
\hline
\end{tabular}

*: Note that the column "average" and the row "total error" don't always add up arithmetically with the data of the table, as a result of rounding up, and that the sum of the ACC with the total error rate does not always equals $100 \%$ because of absence of minor errors for piperacillin/tazobactam, changing the proportions of error rates.

\section{DISCUSSION}

In order to assess the accuracy of Vitek 2 in the present setting, one can first perform an analysis by clinical category $(\mathrm{R} / \mathrm{I} / \mathrm{S})$. Overall, the rate of agreement between both methods was $91.15 \%$. This means that there was a discrepancy of some kind between the results obtained with Vitek 2 and those obtained with 
A COMPARISON WITH ETEST RESULTS.

Table 4. Distribution of similar versus variable differences in clinical categories among centres (in absolute numbers out of a total of 150 strains).

\begin{tabular}{|c|c|c|c|c|c|c|}
\hline & Amikacin & Cefepime & Ceftazidime & Ciprofloxacin & Meropenem & $\begin{array}{c}\text { Piperacillin/ } \\
\text { tazobactam }\end{array}$ \\
\hline Similar in all 3 & 1 & 2 & 2 & 2 & - & 2 \\
\hline Variable & 7 & 6 & 8 & 1 & 5 & 2 \\
\hline Only in one & 14 & 33 & - & 13 & 12 & 16 \\
\hline
\end{tabular}

Etest in only $8.85 \%$. Amongst these, one can distinguish between the so-called minor errors $(8.05 \%$ in this study), whereby the results were within a category which was susceptible or resistant with one method and intermediate with the other method; major errors $(0.57 \%)$ whereby the reference method indicates susceptibility and the test method resistance; and finally, very major errors $(1.57 \%)$ defined as resistance with the reference method and susceptibility with the test method. ${ }^{18}$ Rationale behind this classification is that one does not want to treat patients with a potentially inactive agent, in case the reference method would yield "resistance" whereas the test method would suggest susceptibility.

However, strictly speaking, these terms have been coined in studies using broth microdilution as the reference method. In the present study though, Etest and not broth microdilution, played the role of method of reference. As such, the terms minor, major and very major errors could be of some impropriety.

An overall category error rate of $<10 \%$ has been established as a standard of performance for susceptibility tests. Included in this target percentage are very major error rates of $\leq 1.5 \%$ and major error rates of $\leq$ $3 \%$. 519 The pooled findings in this study complied with this expectation, except for a small excess of $1.57 \%$ very major errors. Elder recommended a combined major-minor error rate of $<7 \%$, which is only achieved for ciprofloxacin, meropenem and piperacillin/tazobactam, when antibiotics were considered separately.

If one considers the resistance rate expressed in clinical categories (see Table 1, row 2-4), $15 \%$ of $P$. aeruginosa isolates were found non-susceptible (resistant plus intermediate) to amikacin, meropenem and piperacillin/tazobactam, 20\% to cefepime and ceftazidime, but in the case of ciprofloxacin this rate reached almost $35 \%$. These values are in agreement with the susceptibility pattern of this organism in Belgium. ${ }^{20}$

When one analyses the concordance between both methods in terms of clinical categories according to the antibiotic tested (as in Table 1, row 5-9), the Vitek 2 system appears to agree rather well with the Etest for most antibiotics (ACC over 90\% conform Jorgensen's criteria, major and very major errors within the limits), except for cefepime (84.58\% ACC) and for piperacillin/tazobactam (6.20\% very major errors). In the case of cefepime, there were $1.50 \%$ very major errors, but the $13.71 \%$ minor errors were responsible for the largest portion of discrepancies between both methods. Chandler also reported of a $12.5 \%$ minor error rate for cefepime comparing Vitek 2 with disk diffusion and broth microdilution. ${ }^{12} \mathrm{He}$ did not register very major errors for cefepime or piperacillin/tazobactam though. It is interesting to remark that minor errors can occur as a result of one dilution difference, the experimental error on the method. Vitek has shown routinely to produce more resistant results than broth microdilution for cefepime. Jones also compared Vitek versus Etest results for cefepime, with $18.3 \%$ minor errors and 3.3\% major errors. He recommends Vitek users to use broth microdilution, disk diffusion or Etest to appropriately guide infection chemotherapy for gram-negative strains. ${ }^{13}$ The Vitek system (Vitek Card GNS-116) produced a high major error rate of $8.9 \%$ for meropenem compared with the broth microdilution reference method. ${ }^{2}$ On the other hand, Etest MICs for meropenem have shown to be significantly higher than broth microdilution MICs for $P$. aeruginosa isolates. ${ }^{2}$ Similarly, Etest has recently been reported to yield more resistant results than other methods (e.g. broth dilution) when testing $P$. aeruginosa for susceptibility to cefepime. ${ }^{21}$

In our study, Etest for cefepime showed in $6.85 \%$ (data not shown) of all strains more than one dilution greater MICs than Vitek, explaining the very major errors.

In the case of piperacillin/tazobactam, Table 1 shows a very major error rate of $6.20 \%$. One of the reasons for 
this finding could be the absence of an "intermediate" category. Indeed, the NCCLS standards for this agent have been modified on several occasions in the last 2 decades and the present susceptibility breakpoint for $P$. aeruginosa is different from that of other Enterobacteriaceae $(64 \mu \mathrm{g} / \mathrm{ml}$ instead of $32 \mu \mathrm{g} / \mathrm{ml}) .{ }^{15}$ Sensititre was concordant with Etest in 6 of the 7 strains with piperacillin/tazobactam very major errors and in the only very major error with cefepime. Sensititre taken as consensus makes us suggesting that Etest and not Vitek 2 is more correct.

Note, however, that the MIC ranges differed between both methods. As was detailed in the section materials and methods, the lowest and the highest available concentration of Etest exceeded those of Vitek 2. For that reason, all calculations were done after the ranges of both tests had been rendered identical. This means all results of Etest below or beyond the Vitek 2 range were considered equal to the lowest or highest (respectively) Vitek 2 dilution available.

In order to explain the few differences observed, one could compare the results obtained in every particular centre, to make sure the overall results apply locally on the one hand, and in an effort to explain the less favourable results in terms of the low ACC for cefepime and the very major errors for piperacillin/tazobactam.

Table 3 indicates that all three centres performed roughly as well, overall, with an ACC exceeding $90 \%$ and with a very major error rate slightly augmented in two centres.

Discrepancies between methods can be inherent to the methods themselves, or they can be the result of some interpretative problems by the laboratory personnel and/or the reporting microbiologist. For this reason, table 4 analyses the repetitive character of discrepancies. It shows that the same difference in clinical error categories only was found in a very limited number of cases in all three participating laboratories: never with meropenem, once with amikacin and twice with the other antibiotics. In those cases, one might conclude both methods yielded somewhat different results. In instances with variable patterns of differences between the individual laboratories, one might be tempted to think they were due to human "errors" rather than to intrinsic methodological differences.

In conclusion: the 150 clinical strains studied in this report exhibited a susceptibility pattern similar to other recent collections in Belgium, irrespective of the testing method. By and large, Vitek 2 showed high concordance with Etest, meeting the criteria for accuracy.
Some differences did occur in ACC for cefepime. Very major errors were registered in excess for piperacillin/ tazobactam. They seem to be due to some difficulty in interpreting Etest in individual centres. The same error very rarely occurred in the 3 centres. The overall accuracy of the Vitek 2 system exceeded $91 \%$, with only cefepime not reaching the acquired $90 \%$ of ACC (84.58\%).

Vitek 2 appears to yield valuable information to the clinician concerning the antimicrobials amikacin, ceftazidime, ciprofloxacin and meropenem, in the setting of $P$. aeruginosa infection. For cefepime, and piperacillin/tazobactam, a confirmatory testing by means of disk diffusion is worth considering.

\section{ACKNOWLEDGEMENT}

This study was granted by the Fund for Scientific Research-Flanders.

\section{SAMENVATTING}

Pseudomonas aeruginosa infecties bij ernstig verzwakte patiënten vereisen accurate antimicrobiële gevoeligheidsgegevens, aangezien hun behandeling voornamelijk gebaseerd is op antibiotische efficiëntie. Vitek 2, een populaire geautomatiseerde gevoeligheidsmethode, werd vergeleken met Etest om zijn betrouwbaarheid te evalueren op 150 Belgische $P$. aeruginona isolaten.

Vitek 2 en Etest vertoonden een hoge mate van concordantie. Discrepanties in klinische categorie werden vastgesteld met cefepime (meer mineure fouten en maximaal toegelaten percentage zeer grote fouten) en met piperacilline/tazobactam (meer zeer grote fouten). Bij pseudomonas infecties blijkt Vitek 2 de clinicus waardevolle informatie te leveren voor de antibiotica amikacine, ceftazidime, ciprofloxacine en meropenem. Voor cefepime en piperacilline/tazobactam valt een bijkomende confirmerende disk diffusie test te overwegen. 


\section{REFERENCES}

1. Rello J, Jubert P, Valles J et al. Evaluation of outcome for intubated patients with pneumonia due to Pseudomonas aeruginosa. Clin Inf Dis. 1996; 23: 973-8.

2. Steward C, Mohammed J, Swenson J et al. Antimicrobial Susceptibility Testing of Carbapenems: Multicenter Validity Testing and Accuracy Levels of Five Antimicrobial Test Methods for Detecting Resistance in Enterobacteriaceae and Pseudomonas aeruginosa Isolates. J Clin Microbiol. 2003; 41(1): 351-8.

3. Celis R, Torres A, Gatell JM et al. Nosocomial pneumonia: a multivariate analysis of risks and prognosis. Chest 1988; 93: 318-24.

4. Torres A, Aznar R, Gatell JM et al. Incidence, risks, and prognosis factors of nosocomial pneumonia in mechanically ventilated patients. Am Rev Respir Dis. 1990; 142: 523-8.

5. Doern G, Brueggemann A, Perla R et al. Multicenter laboratory evaluation of the bioMerieux Vitek antimicrobial susceptibility testing system with 11 antimicrobial agents versus members of the family Enterobacteriaceae and Pseudomonas aeruginosa. $J$ Clin Microbiol. 1997; 35(8): 2115-9.

6. Barry J, Brown A, Ensor V et al. Comparative evaluation of the Vitek advanced expert system (AES) in five UK hospitals. $J$ Antimicrob Chemother. 2003; 51: 1191-202.

7. Livermore D, Struelens M, Amorim J et al. Multicenter evaluation of the Vitek 2 advanced expert system for interpretive reading of antimicrobial resistance tests. J Antimicrob Chemother. 2002; 49: 289-300.

8. Leclercq R, Nicolas-Chanoine M-H, Nordmann $\mathrm{P}$ et al. Multicenter Evaluation of an Automated System Using Selected Bacteria That Harbour Challenging and Clinically Relevant Mechanisms of Resistance to Antibiotics. Eur J Clin Microbiol Infect Dis. 2001; 20: 626-35.

9. Cantón R, Perez-Vazquez M, Oliver A et al. Validation of the Vitek 2 and the advance expert system with a collection of Enterobacteriaceae harbouring extended spectrum or inhibitor resistant B-lactamases. Diagn Microbiol Infect Dis. 2001; 41: 65-70.

10. Karlowsky J, Weaver M, Thornsberry C, Dowzicky M, Jones M, Sahm D. Comparison of Four Antimicrobial Susceptibility Testing Methods To Determine the In Vitro Activities of Piperacillin and Piperacillin-Tazobactam against Clinical Isolates of Enterobacteriaceae and Pseudomonas aeruginosa. J Clin Microbiol. 2003; 41(7): 3339-43.
11. Burns J, Saiman L, Whittier S et al. Comparison of two commercial systems (Vitek and MicroScan WalkAway) for antimicrobial susceptibility testing of Pseudomonas aeruginosa isolates from cystic fibrosis patients. Diagn Microbiol Infect Dis. 2001; 39: 257-260.

12. Chandler LJ, Poulter M, Reisner B, Woods G. Clinical evaluation of the Vitek automated system with cards GNS 122 and 127 and VTK-R07.01 software for antimicrobial susceptibility testing of Pseudomonas aeruginosa. Diagn Microbiol Infect Dis. 2002; 42: 71-3.

13. Jones R, Biedenbach D, Marshall S, Pfaller M, Doern G. Evaluation of the Vitek System to Acurrately Test the Susceptibility of Pseudomonas aeruginosa Clinical Isolates Against Cefepime. Diagn Microbiol Infect Dis. 1998; 32: 107-10.

14. Jones R, Marshall S, Zerva L. Critical Evaluation of the Vitek GNS-F6 Card Results Compared to Standardized, Reference Susceptibility Test Methods. Diagn Microbiol Infect Dis. 1997; 28: 35-40.

15. Doern G, Vautour R, Gaudet M, Levy B. Clinical Impact of Rapid In Vitro Susceptibility Testing and Bacterial Identification; J Clin Microbiol. 1994; 32: 1757-62.

16. Biedenbach DJ, Jones RN. Interpretive Errors Using an Automated System for the Susceptibility Testing of Imipenem an Aztreonam. Diagn Microbiol Infect Dis. 1995; 21: 57-60.

17. National Committee for Clinical Laboratory Standards. Methods for dilution antimicrobial susceptibility tests for bacteria that grow aerobically - Sixth Edition: Approved standard M7A6, NCCLS, Villanova, PA, USA (2003).

18. Jorgensen J \& Ferraro M. Antimicrobial susceptibility testing: general principles and contemporary practices. Clin Infect Dis. 1998; 26: 973-80.

19. Elder B. Verification and Validation of Procedures in the Clinical Microbiology Laboratory. Clinical microbiology newsletter 1997; 19(20): 153-60.

20. Glupczynski Y, Delmée M, Goossens H et al. Distribution and Prevalence of antimicrobial resistance among Gram-negative isolates in intensive care units (ICU) in Belgian hospitals between 1996 and 1999. Acta Clin Belg. 2001; 56(5): 297-306.

21. Schwebke J, Waites K, Beverly A et al. Inaccuracy of automated susceptibility testing for cefepime against Pseudomonas aeruginosa. In Program and Abstracts of the Forty third Interscience Conference on Antimicrobial Agents and Chemotherapy, Chicago, Il, 2003. Abstract D-234, p. 174. American Society for Microbiology, Washington, DC, USA. 